\title{
FOXM1 participates in PLK1-regulated cell cycle progression in renal cell cancer cells
}

\author{
ZHE ZHANG $^{1}$, GUOJUN ZHANG ${ }^{2}$ and CHUIZE KONG ${ }^{1}$ \\ ${ }^{1}$ Department of Urology, The First Hospital of China Medical University, Shenyang, Liaoning 110001; \\ ${ }^{2}$ Department of Hematology, Shengjing Hospital of China Medical University, Shenyang, Liaoning 110022, P.R. China
}

Received February 26, 2015; Accepted February 4, 2016

DOI: $10.3892 / \mathrm{ol} .2016 .4228$

\begin{abstract}
The regulation of entry into and progression through mitosis is important for cell proliferation. Polo-like kinase 1 (PLK1) is involved in multiple stages of mitosis. Forkhead box protein M1 (FOXM1) has multiple functions in tumorigenesis and, in elevated levels, is frequently associated with cancer progression. The present study reports that FOXM1, a substrate of PLK1, controls the transcription mechanism that mediates the PLK1-dependent regulation of the cell cycle. The present study investigated the expression of PLK1 and FOXM1 in the clear renal cell carcinoma 769-P and ACHN cell lines, and indicated that the expression of PLK1 and FOXM1 are correlated in human renal cell cancer cell lines and that the suppression of PLK1 may decrease the expression of FOXM1. The knockdown of FOXM1 or PLK1 in renal cell cancer cell lines caused cell cycle progression to be blocked. As a result, the present study indicated the involvement of FOXM1 in PLK1-regulated cell cycle progression.
\end{abstract}

\section{Introduction}

Renal cell carcinoma possesses the highest mortality rate of the genitourinary cancers, and is a heterogeneous disease that shows molecular and genetic heterogeneity and complexity (1). Clear cell renal cell carcinoma is the most common histological subtype of renal cell carcinoma, accounting for $\sim 80 \%$ of cases of renal tumors (2). Early diagnosis and medical intervention are vital in decreasing mortality rates and promoting quality of life. In addition, novel molecular markers for kidney cancer are urgently required, as markers may aid the evaluation of individual risks, the prediction of patient outcomes, prognoses and therapeutic effects of treatment, and the promotion of personalized treatment.

Correspondence to: Professor Zhe Zhang, Department of Urology, The First Hospital of China Medical University, 155 Nanjing North Street, Shenyang, Liaoning 110001, P.R. China E-mail: zhangzheurology@gmail.com

Key words: renal cancer, polo-like kinase 1, cell proliferation, forkhead box protein M1, cell cycle
Polo-like kinase 1 (PLK1) is one of three isoforms of PLK, the others being PLK2 and PLK3, and has been found to be overexpressed in several human cancers (3). The serine/threonine kinase PLK1 governs several mitotic stages, including entry into mitosis, centrosome maturation, bipolar spindle assembly, activation of the anaphase-promoting complex/cyclosome by phosphorylation of early mitotic inhibitor 1 , chromosome segregation and mitotic exit (4). The overexpression of PLK1 is associated with a poor prognosis in a variety of cancers (5-9). Beyond the regulation of mitosis, PLK1 possesses cancer cell-specific functions in G1/S transition and DNA replication $(10,11)$. Previous studies have indicated that PLK1 overexpression may not simply be a consequence of enhanced proliferation, and that PLK1 is likely to actively contribute to early carcinogenesis $(12,13)$.

The mammalian transcription factor forkhead box protein M1 (FOXM1) is important in regulating mitotic entry and the subsequent execution of the mitotic program by controlling the expression of a cluster of G2/M target genes (14). Previous studies have indicated that, during the embryonic period, FOXM1 is overexpressed in all tissues (15); however, in adulthood, FOXM1 expression only occurs in the tissues with active proliferation and metabolism (16). In various types of tumor cells, FOXM1 is highly expressed in the nucleus and cytoplasm, and causes the dysfunction of regulation processes (17-21). In particular, FOXM1 controls mitotic entry by the periodic upregulation of a group of genes that are maximally expressed as cells progress through the late G2 and into the M phase (14). $\mathrm{Fu}$ et al indicated that PLK1 regulates FOXM1 transcriptional activity by direct phosphorylation, and therefore, controls the execution of the transcriptional program required for mitotic progression (22). The study by Fu et al established a novel association between PLK1 and the key mitotic transcription factor FOXM1, and provided evidence as to how PLK1 globally regulates cell division (22). However, the precise mechanism by which PLK1-dependent phosphorylation enhances FOXM1 transcriptional activity remains to be determined.

The present study investigated the expression of PLK1 and FOXM1 in the clear renal cell carcinoma 769-P and ACHN cell lines in order to examine the coordinate association between the two proteins. The knockdown of FOXM1 or PLK1 in renal cell cancer cell lines caused cell cycle progression to be blocked. In addition, the involvement of FOXM1 in PLK1-regulated cell cycle progression was also indicated. 


\section{Materials and methods}

Cell lines and reagents. The renal cell cancer 769-P and ACHN cell lines were purchased from the Chinese Academy of Sciences Cell Bank (Shanghai, China). All cell lines were carefully maintained in a humidified culture incubator at $37^{\circ} \mathrm{C}$ in $5 \% \mathrm{CO}_{2}$. The cell lines were grown in RPMI 1640 (Invitrogen; Thermo Fisher Scientific, Waltham, MA, USA) supplemented with $10 \%$ fetal bovine serum (Invitrogen; Thermo Fisher Scientific).

Antibodies against PLK1 (mouse anti-human monoclonal antibody; dilution, 1:2,000; catalog no., ab17056) and FOXM1 (rabbit anti-human polyclonal antibody; dilution, 1:2,000; catalog no., ab180710) were purchased from Abcam (Cambridge, MA, USA). Antibodies specific for cyclin B1 (rabbit anti-human monoclonal antibody; dilution, 1:2,000; catalog no., \#12231), aurora B (rabbit anti-human monoclonal antibody; dilution, 1:2,000; catalog no.,\#3094) and glyceraldehyde-3-phosphate dehydrogenase (GAPDH; rabbit anti-human monoclonal antibody; dilution, 1:2,000; catalog no., \#5174) were obtained from Cell Signaling Technology, Inc. (Danvers, MA, USA). V-akt murine thymoma viral oncogene homolog 1 (AKT1; mouse anti-human monoclonal antibody; dilution, 1:2,000; catalog no., SC-55523) and ribosomal protein S6 kinase, $70 \mathrm{kDa}$, polypeptide 1 (P70S6K1; rabbit anti-human polyclonal antibody; dilution, 1:2,000; catalog no. SC-230) antibodies were obtained from Santa Cruz Biotechnology, Inc. (Dallas, TX, USA).

Total RNA extraction, cDNA synthesis and reverse transcription-quantitative polymerase chain reaction $(R T-q P C R)$. Total RNA was prepared by using Invitrogen TRIzol (Thermo Fisher Scientific), and 500 ng of total RNA was reverse transcribed into cDNA using the PrimeScript ${ }^{\mathrm{TM}}$ RT Master Mix (Perfect Real Time; Takara Biotechnology Co. Ltd., Dalian, China). RT-qPCR was performed under the following conditions: $95^{\circ} \mathrm{C}$ for $15 \mathrm{sec}$ and $60^{\circ} \mathrm{C}$ for $1 \mathrm{~min}$, for 35 cycles, with an initial denaturation at $95^{\circ} \mathrm{C}$ for $10 \mathrm{~min}$. The expression of the housekeeping gene $\beta$-actin was examined as a control. The primer sequences are shown in Table I. The relative expression value of the target gene and $\beta$-actin was calculated. The experiments were conducted in triplicate.

Western blot analysis. Frozen cells were washed twice with ice-cold phosphate-buffered saline (PBS) and homogenized on ice in $10 \mathrm{vol}(\mathrm{wt} / \mathrm{vol})$ of lysis buffer containing $20 \mathrm{mM}$ Tris- $\mathrm{HCl}, 1 \mathrm{mM}$ ethylenediaminetetraacetic acid, $50 \mathrm{mM} \mathrm{NaCl}$, $50 \mathrm{mM} \mathrm{NaF}, 1 \mathrm{mM} \mathrm{Na} \mathrm{VO}_{4}, 1 \%$ Triton-X100, $1 \mathrm{mM}$ phenylmethanesulfonyl fluoride and phosphatase inhibitor, using a Diax 900 homogenizer (Heidolph, Schwabach, Germany). The homogenate was centrifuged at $10,000 \times \mathrm{g}$ for $30 \mathrm{~min}$ at $4^{\circ} \mathrm{C}$. The supernatant was collected and stored at $-80^{\circ} \mathrm{C}$. The protein content was determined using a bicinchoninic acid protein assay kit (Pierce Biotechnology, Inc., Rockford, IL, USA). From each sample preparation, $70 \mu \mathrm{g}$ of total protein was separated using $8 \%$ sodium dodecyl sulfate-polyacrylamide gel electrophoresis (SDS-PAGE), and the bands were then transferred to polyvinylidene fluoride blot membranes (EMD Millipore, Billerica, MA, USA). The total protein extracts were analyzed by immunoblotting with indicated antibodies following the SDS-PAGE analysis. Immunoblotting was performed using mouse monoclonal primary antibodies specific for PLK1 and GAPDH and rabbit primary antibodies specific for cyclin B1, aurora B and FOXM1 (Abcam, Hong Kong, China). Subsequent to blocking the non-specific binding sites with $5 \%$ bovine serum albumin (Amresco, Solon, OH, USA) in Tris-buffered saline ( $\mathrm{pH} 7.5$; Amresco) containing $0.05 \%$ Tween-20 (Amresco) (TBST), the PLK1, FOXM1, cyclin B1, AKT1, P70S6K1, GAPDH and aurora B1 primary antibodies were incubated on the membranes for overnight at $4^{\circ} \mathrm{C}$ in TBST. Following 3 washes in TBST, the membranes were incubated for $2 \mathrm{~h}$ at $37^{\circ} \mathrm{C}$ with goat polyclonal secondary antibodies against mouse or rabbit IgG (dilution, 1:5,000; Abcam) labeled with horseradish peroxidase. The proteins were detected using an enhanced chemiluminescence detection system (Pierce ECL Plus Western Blotting Substrate; Pierce Biotechnology, Inc.), according to the manufacturer's protocol. Specific bands for the target protein were identified using a MBI Fermentas prestained protein molecular weight marker (Thermo Fisher Scientific). The EC3 Imaging System (Ultra-Violet Products, Ltd., Cambridge, UK) was used to capture images of the specific bands, and the optical density of each band was measured using Image $\mathbf{J}$ software. The ratio between the optical densities of target proteins of the same sample was calculated as relative content and expressed graphically.

Knockdown of PLK1 and FOXM1. Double-stranded small interfering (si)RNA oligomers were transfected into 769-P and ACHN cells using Lipofectamine ${ }^{\circledR} 2000$ Transfection Reagent (Thermo Fisher Scientific), according to the manufacturer's protocol. Briefly, cells were seeded into 6-well plates at a density of 100,000 cells per well and grown for $12 \mathrm{~h}$ prior to transfection with human PLK1 siRNA, 5'-CCCUCA CAGUCCUCAAUAATT-3', or human FOXM1 siRNA, 5'-GGAAATGCT TGTGATTCAACA-3' (Thermo Fisher Scientific), for 24 or $48 \mathrm{~h}$. The Invitrogen negative siRNA control was purchased from Thermo Fisher Scientific.

Plasmid construction and transfection. ACHN cells were transfected with the PLK1 pEX-2-PLK1 expression plasmid (Shanghai Genepharma Co., Ltd, Shanghai, China) using Invitrogen Lipofectamine ${ }^{\circledR} 2000$ (Thermo Fisher Scientific). The expression of transfected genes was confirmed using western blot analysis.

Analysis of the cell cycle distribution. The 769-P and ACHN cells, with or without transfection, were plated in $25 \mathrm{~cm}^{2}$ flasks and incubated overnight. Briefly, the attached cells were trypsinized and collected by centrifugation $(1,000 \mathrm{x} \mathrm{g})$, washed in PBS and fixed in cold $70 \%$ ethanol for $1.5 \mathrm{~h}$ at $4^{\circ} \mathrm{C}$. Subsequent to fixation, the cells were washed in PBS again and centrifuged for $5 \mathrm{~min}$ at $800 \mathrm{x}$ g. The PBS was discarded and propidium iodide (BD Biosciences, Franklin Lakes, NJ, USA) was added to a final concentration of $50 \mu \mathrm{g} / \mathrm{ml}$, which was stored in the dark at $4^{\circ} \mathrm{C}$ for $30 \mathrm{~min}$. Flow cytometric analysis was performed on the FACSCalibur flow cytometer (BD Biosciences, Oxford, UK). Finally, the cell cycle was analyzed using Cell Quest software (BD Biosciences).

Statistical analysis. SPSS version 17.0 (SPSS, Inc., Chicago, IL, USA) was used to perform data analysis. Student's $t$-test 
Table I. The primer sequences of polo-like kinase 1, forkhead box protein M1 and $\beta$-actin.

\begin{tabular}{llr}
\hline Gene name & Forward primer (5'-3') & Reverse primer $\left(5^{\prime}-3^{\prime}\right)$ \\
\hline Polo-like kinase 1 & CAAGAAGAATGAATACAGTA & GGATATAGCCAGAAGTAA \\
Forkhead box protein M1 & AGCAGTCTCTTACCTTCC & CTGGCAGTCTCTGGATAA \\
$\beta$-actin & TAATCTTCGCCTTAATACTT & AGCCTTCATACATCTCAA
\end{tabular}

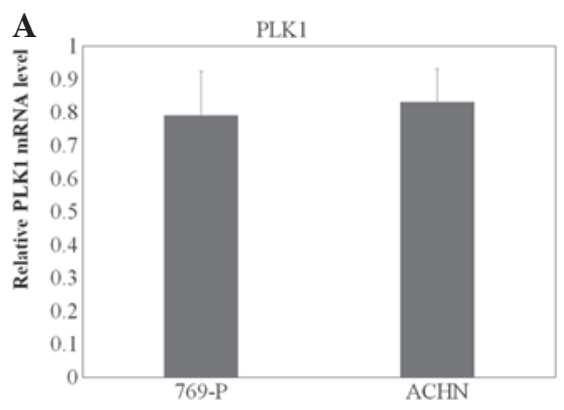

B

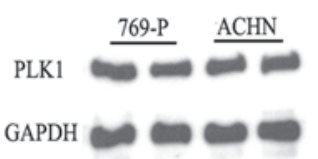

D

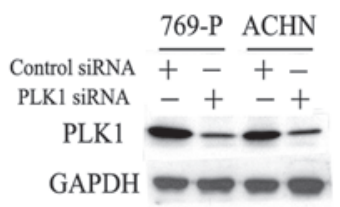

E

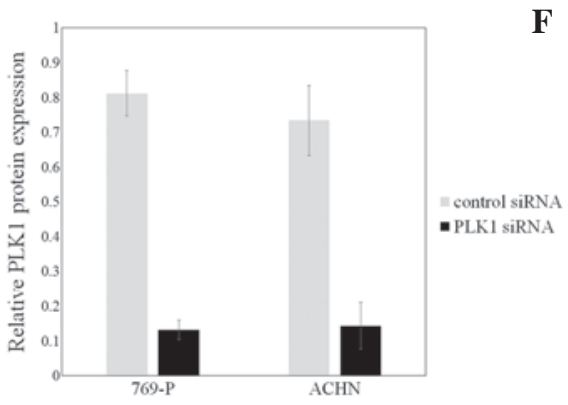

$\mathbf{F}$
C
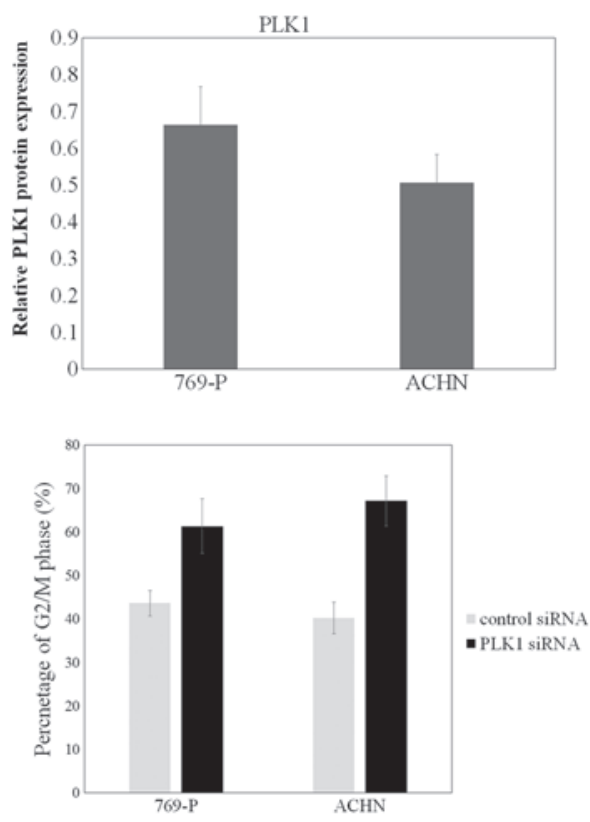

Figure 1. Expression of PLK1 in renal cell cancer 769-P and ACHN cell lines. The knockdown of PLK1 may induce cell cycle arrest in human renal cell cancer cell lines. (A) Ratio between the cycle threshold value of PLK1 and GAPDH of the same group, determined by reverse transcription-quantitative polymerase chain reaction was calculated and expressed graphically. The data were representative of 3 individual experiments. (B) Band intensities indicate the expression of PLK1 in 769-P and ACHN cells, using western blot analysis. GAPDH was used as a loading control to assure equal amounts of protein in all lanes. (C) Ratio between the optical density of PLK1 and GAPDH of the same sample was calculated and expressed graphically. The data are representative of 3 individual experiments. (D) Band intensities indicate the expression of PLK1 with treatment of PLK1 siRNA. (E) Ratio between the optical density of PLK1 and GAPDH of the same sample was calculated and expressed graphically. (F) Cell cycle was analyzed using flow cytometry with PLK1 siRNA. mRNA, messenger RNA; siRNA, small interfering RNA; PLK1, polo-like kinase 1; GAPDH, glyceraldehyde 3-phosphate dehydrogenase.

was used to analyze the data from the RT-qPCR and western blot analysis of the cells. Groups with or without treatment were compared using one-way analysis of variance. The Pearson product-moment correlation coefficient was used to determine the correlation between mRNA and protein expression. $\mathrm{P}<0.05$ was considered to indicate a statistically significant difference.

\section{Results}

Knockdown of PLK1 may induce cell cycle arrest in human renal cell cancer cell lines. To identify the role of PLK1 in human renal cell cancer cells, the expression of PLK1 was examined by RT-qPCR and western blot analysis in 2 human renal cell cancer cell lines, 769-P and ACHN. The mRNA and protein expression of PLK1 was high and stable in the 2 cell lines (Fig. 1A-C). Then, a specific effective siRNA for PLK1 was transfected to downregulate the expression of PLK1 (Fig. 1D and E). The effect of PLK1 knockdown on the cell cycle was then examined. The flow cytometer was used to analyze the cell cycle. The results revealed that the 769-P and ACHN cells transfected with PLK1 siRNA demonstrated increased percentages of cells (61.39 and 67.17\%, respectively) in the G2/M phase. Additionally, in the mock (without any additions) and control siRNA groups, the numbers of cells in the G2/M phase were significantly decreased compared with the PLK1 siRNA group (Fig. 1F).

Downregulation of FOXM1 may block cell cycle progression in human renal cell cancer cell lines. FOXM1 was previously demonstrated to be upregulated in bladder cancer tissues and cells, compared with normal bladder tissues and cells (23). In the present study, FOXM1 was also highly expressed in 769-P and ACHN cells (Fig. 2A-C). Then, a specific effective siRNA for FOXM1 was transfected to decrease the expression of FOXM1 (Fig. 2D and E). To confirm the role of FOXM1 in cell division, the cell cycle distribution of FOXM1 siRNA-treated 769-P and ACHN cells was examined. As shown in Fig. 2F, elevated cell numbers in the G2/M phase of the cell cycle were indicated following FOXM1 siRNA transfection, compared with the mock group and control siRNA group. 


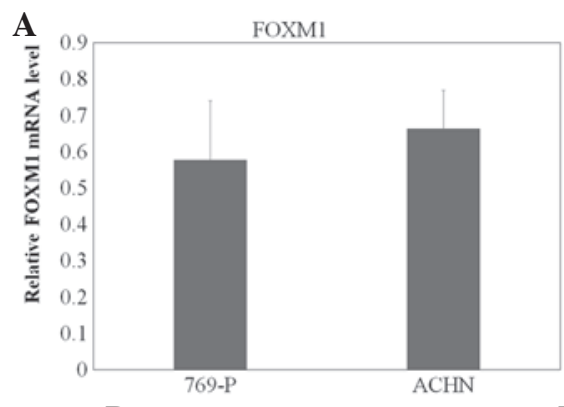

D

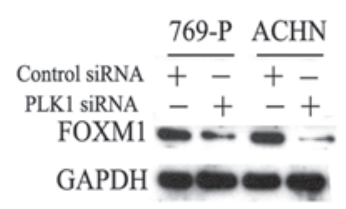

B

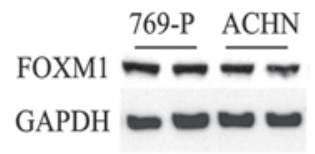

$\mathbf{E}$

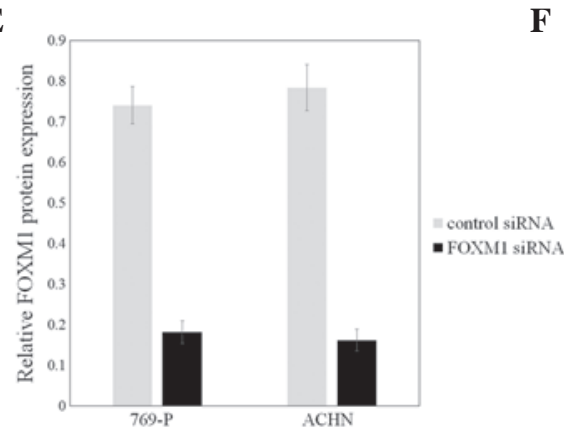

C

F
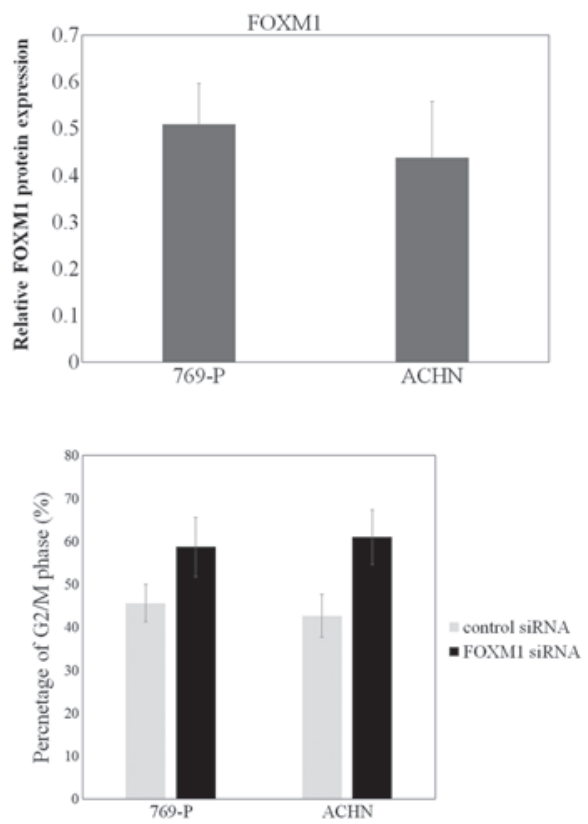

Figure 2. Expression of FOXM1 in renal cell cancer 769-P and ACHN cell lines. The knockdown of FOXM1 may induce cell cycle arrest in human renal cell cancer cell lines. (A) Ratio between the cycle threshold value of FOXM1 and GAPDH of the same group by reverse transcription-quantitative polymerase chain reaction was calculated and expressed graphically. The data were representative of 3 individual experiments. (B) Band intensities indicate the expression of FOXM1 in 769-P and ACHN cells, using western blot analysis. GAPDH was used as a loading control to assure equal amounts of protein in all lanes. (C) Ratio between the optical density of FOXM1 and GAPDH of the same sample was calculated and expressed graphically. The data are representative of 3 individual experiments. (D) Band intensities indicate the expression of FOXM1 with the treatment of FOXM1 siRNA. (E) Ratio between the optical density of FOXM1 and GAPDH of the same sample was calculated and expressed graphically. (F) Cell cycle was analyzed using flow cytometry with FOXM1 siRNA. mRNA, messenger RNA; siRNA, small interfering RNA; FOXM1, forkhead box protein M1; GAPDH, glyceraldehyde 3-phosphate dehydrogenase.

Expression of PLK1 and FOXM1 is correlated in human renal cell cancer cell lines and the suppression of PLKI may decrease the expression of FOXM1. The aforementioned findings suggested that PLK1 and FOXM1 were involved in cell cycle regulation in renal cell cancer cells. Therefore, an association may exist between PLK1 and FOXM1 in cell cycle progression. To test this hypothesis, Pearson product-moment correlation coefficient was used to determine the correlation between the mRNA and protein expression. The results indicated a significant positive correlation between PLK1 and FOXM1 mRNA and protein levels in 769-P and ACHN cells (Fig. 3A-D).

For additional investigation of the association between PLK1 and FOXM1,FOXM1 expression in PLK1 siRNA-treated 769-P and ACHN cells was detected. The data demonstrated that downregulating PLK1 may decrease the expression of FOXM1 (Fig. 3E-G). However, the knockdown of FOXM1 expression by specific siRNA did not evidently affect the expression of PLK1 (Fig. 3H-J). Therefore, PLK1 may regulate FOXM1, and the knockdown of PLK1 may suppress the expression of FOXM1.

Involvement of FOXM1 in PLK1-regulated cell cycle progression. To confirm the participation of FOXM1 in PLK1-regulated cell cycle progression in renal cell cancer cells, the expression of FOXM1 and associated target genes was examined during PLK1 depletion or overexpression (Fig. 4). In PLK1-depleted ACHN cells, the downregulation of FOXM1 and associated target genes, including cyclin B1 and aurora B, was indicated (Fig. 4A and C). Cyclin B1 and aurora $\mathrm{B}$ are required for mitotic progression. In addition, the overexpression of wild-type PLK1 in PLK1-depleted ACHN cells was examined. The results demonstrated that FOXM1, cyclin B1 and aurora B expression levels in PLK1-depleted ACHN cells with overexpression of wild-type PLK1 were restored to the level of expression in the cells without the treatment of PLK1 siRNA (Fig. 4A and C). Next, wild-type PLK1 was transfected into 769-P cells, and the expression of FOXM1, cyclin B1 and aurora B was found to be increased (Fig. 4B and D). In addition, FOXM1 was knocked down by target siRNA in wild-type PLK1 769-P cells. The downregulation of FOXM1 induced the decreased expression of FOXM1, cyclin B1 and aurora B (Fig. 4B and D). Overall, the data indicated that PLK1 regulated FOXM1 and target cycle-associated genes to control mitotic progression.

\section{Discussion}

Previously, major insights into the molecular mechanisms of renal tumorigenesis have emerged from genetic and molecular analyses of families with hereditary benign and malignant kidney tumors. However, the possible heterogeneity of kidney tumors may make the identification of specific markers more challenging. A previous study revealed that PLK1 is overexpressed in renal cancer and participates in the proliferation and invasion of renal cancer cells (8). Although the evidence has elucidated the importance of PLK1 as a tumor promoter in renal cell carcinoma, the precise molecular mechanisms remain largely unknown. In order to better understand the molecular mechanism of PLK1-regulated cell proliferation, gene silencing technology was used in the present study to knock down the expression of PLK1. The data showed that 
A

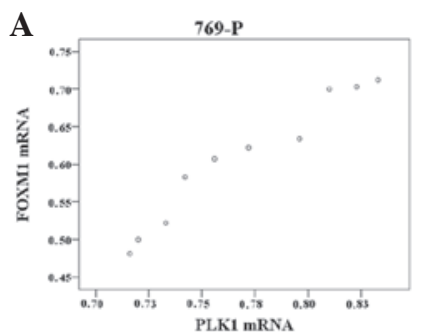

B
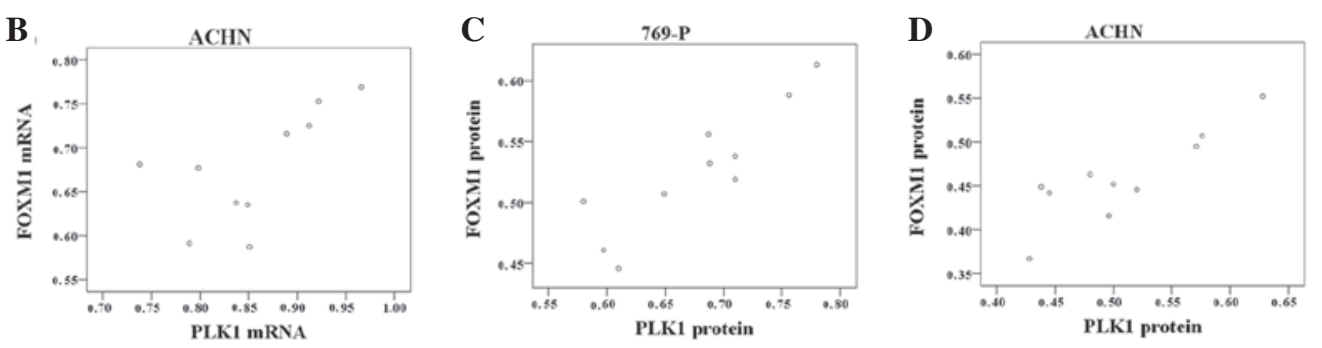

$\mathbf{E}$

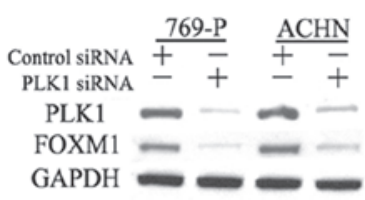

H

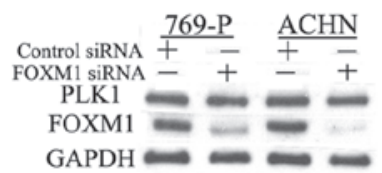

F

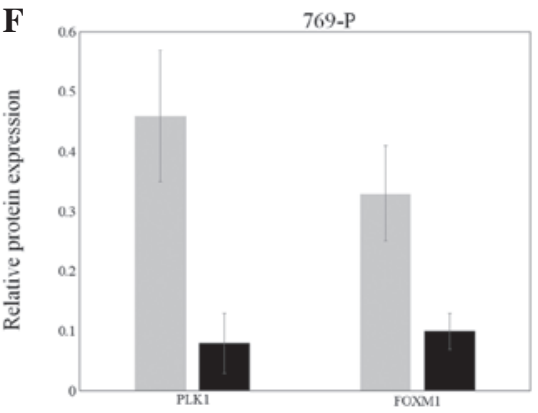

I

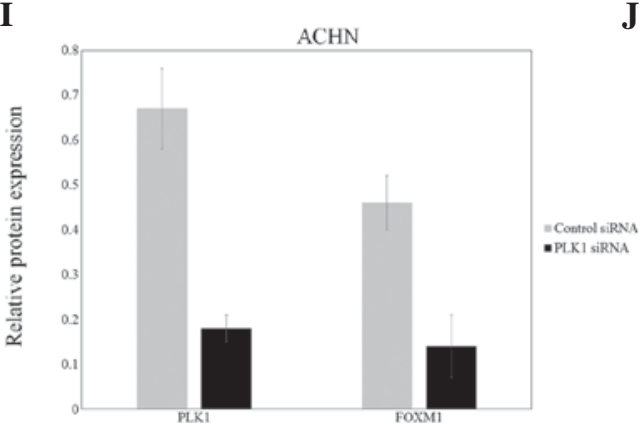

G

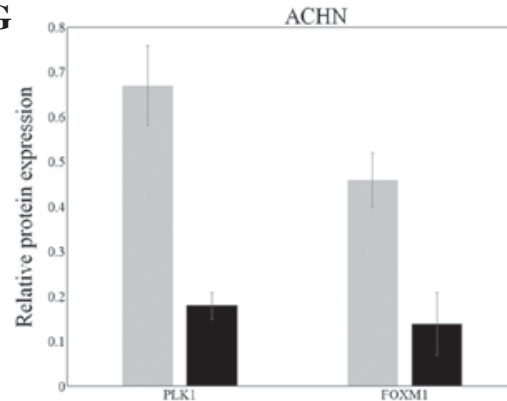

$\mathbf{J}$

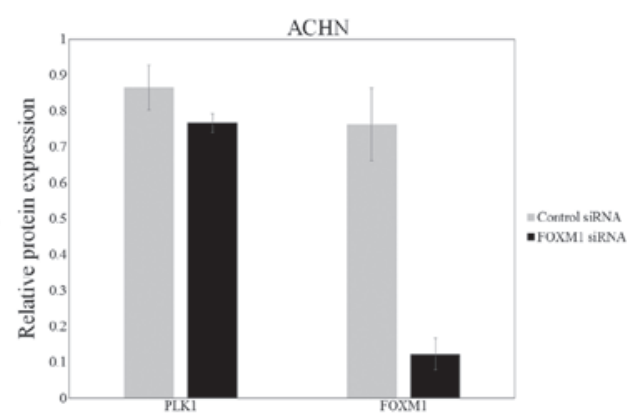

Figure 3. PLK1 and FOXM1 demonstrate a correlation in human renal cell cancer cell lines, and suppressing PLK1 may decrease the expression of FOXM1. Pearson product-moment correlation coefficient was used to determine the correlation of PLK1 and FOXM1 mRNA expression in (A) 769-P and (B) ACHN cells, and PLK1 and FOXM1 protein expression in (C) 769-P and (D) ACHN cells. (E) Band intensities indicate the expression of PLK1 and FOXM1 with treatment of PLK1 siRNA. Ratios between the optical density of target protein and GAPDH of the same tissue using western blot analysis were calculated and expressed graphically in (F) 769-P and (G) ACHN cells. (H) Band intensities indicate the expression of PLK1 and FOXM1 with treatment of FOXM1 siRNA. Ratios between the optical density of target protein and GAPDH of the same tissue using western blot analysis were calculated and expressed graphically in (I) 769-P and (J) ACHN cells. mRNA, messenger RNA; siRNA, small interfering RNA; PLK1, polo-like kinase 1; FOXM1, forkhead box protein M1; GAPDH, glyceraldehyde 3-phosphate dehydrogenase.

A

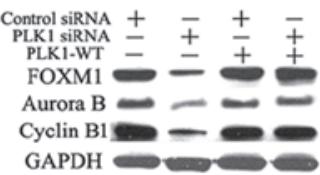

C

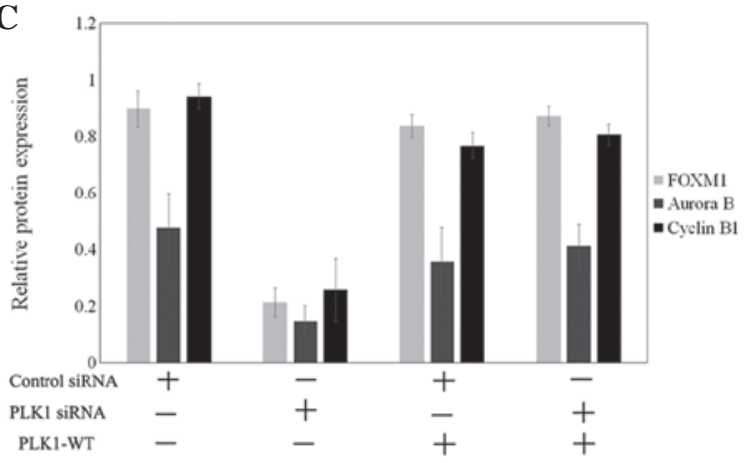

B

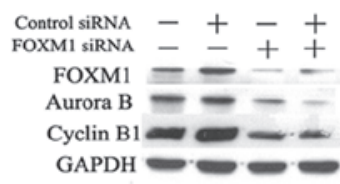

D

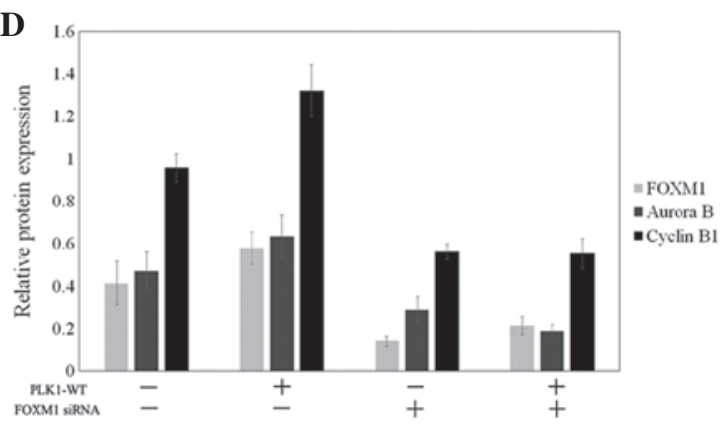

Figure 4. The involvement of FOXM1 in PLK1-regulated cell cycle progression. (A) Band intensities indicate the expression of FOXM1 and FOXM1 target genes with PLK1 depletion or overexpression. (B) Ratio between the optical density of the target protein and GAPDH of the same tissue using western blot analysis was calculated and expressed graphically. (C) Band intensities indicate the expression of FOXM1, Cyclin B1 and Aurora B with treatment of the PLK1-WT into 769-P cells. (D) Ratio between the optical density of target protein and GAPDH of the same tissue using western blot analysis was calculated and expressed graphically. FOXM1, forkhead box protein M1; PLK1, polo-like kinase 1; GAPDH, glyceraldehyde 3-phosphate dehydrogenase, WT, wild type. 
the 769-P and ACHN cells transfected with PLK1 siRNA demonstrated an increased percentage of cells in the G2/M phase, which supports the findings of previous studies $(8,9)$. A previous study reported that PLK1 was important for the proliferation of bladder cancer cells by regulating the cancer cell cycle between the G1/S and G2/M phases (9). Ando et al proposed that the PLK1-mediated phosphorylation of mediator of DNA damage checkpoint 1 is involved in the regulation of G2/M transition (24). Pezuk et al indicated that PLK1 inhibition causes decreased proliferation through cell-cycle arrest, leading to cell death in glioblastoma (25).

Previous studies have revealed that that FOXM1, a substrate of PLK1, controls a transcriptional program that mediates PLK1-dependent regulation of cell-cycle progression (22). Formation of the PLK1-FOXM1 complex allows for the direct phosphorylation of FOXM1 by PLK1 at the G2/M phase and the subsequent activation of FOXM1 activity, which is necessary for the expression of key mitotic regulators, including PLK1 (22). A previous study conducted by Zhang et al revealed a working mechanism by which PLK1 positively regulates the activity and expression levels of FOXM1, which may greatly facilitate therapeutic interventions that focus on targeting the PLK1- or FOXM1-mediated signaling network (26). A study by Wang et al indicated that FOXM1 and PLK1 were significantly associated with certain clinicopathological indices in gallbladder cancer (GC) (27). The evaluation of FOXM1 and PLK1 expression may be an important factor for identifying a poor prognosis of GC (27). FOXM1 was previously demonstrated to be upregulated in bladder cancer tissues and cells, compared with normal bladder tissues and cells (22). The present study demonstrated increased cell numbers in the G2/M phase of the cell cycle following FOXM1 siRNA transfection compared with the mock and control siRNA groups.

To evaluate the association between PLK1 and FOXM1, the Pearson product-moment correlation coefficient was used to determine a significant positive correlation between PLK1 and FOXM1 in 769-P and ACHN cells. In addition, the downregulation of PLK1 was indicated to decrease the expression of FOXM1. However, the knockdown of the FOXM1 protein by specific siRNA did not cause an evident change in PLK1 expression levels. By contrast, a previous study by Sharrocks et al identified that FOXM1 and the associated PLK1 target gene are commonly overexpressed in esophageal adenocarcinomas, and that this association may be extended to other FOXM1 target genes, providing potentially important biomarkers for predicting post-operative disease survival (28). This finding may be due to the tissue-specific presence of FOXM1.

To confirm the participation of FOXM1 in PLK1-regulated cell cycle progression in renal cell cancer cells, the expression of FOXM1 and its associated target genes were assessed during PLK1 depletion or overexpression. The results indicated that PLK1 regulated FOXM1 and the target cycle-associated genes cyclin B1 and aurora B in order to control mitotic progression. This finding provides an updated perspective of the regulation of cell cycle progression by PLK1 for future studies.

The present study identifies FOXM1 as a novel prognostic biomarker for clear cell renal cell carcinoma, and highlights the involvement of FOXM1 in PLK1-regulated cell cycle progression. The results suggest that the knockdown of FOXM1 or PLK1 in renal cell cancer cell lines caused cell cycle progression to be blocked. Therefore, the present study indicated the involvement of FOXM1 in PLK1-regulated cell cycle progression. This hypothesis requires additional studies that analyze the molecular association between the involvement of PLK1 and FOXM1 in the development of clear renal cell carcinoma.

\section{Acknowledgements}

The present study was supported by the National Natural Science Foundation (grant no. 81202000), Liaoning Provincial Natural Science Foundation (grant no. 2013021066), and Shenyang City Project of Key Laboratory (grant no. F13-293-1-00).

\section{References}

1. Tomaszewski JJ, Uzzo RG and Smaldone MC: Heterogeneity and renal mass biopsy: A review of its role and reliability. Cancer Biol Med 11: 162-172, 2014.

2. Farhadi A, Behzad-Behbahani A, Geramizadeh B, Sekawi Z, Rahsaz M and Sharifzadeh S: High-risk human papillomavirus infection in different histological subtypes of renal cell carcinoma. J Med Virol 86: 1134-1144, 2014.

3. van de Weerdt BC and Medema RH: Polo-like kinases: A team in control of the division. Cell Cycle 5: 853-864, 2006.

4. Wang G, Chen Q, Zhang X, Zhang B, Zhuo X, Liu J, Jiang Q and Zhang C: PCM1 recruits PLK1 to the pericentriolar matrix to promote primary cilia disassembly before mitotic entry. J Cell Sci 126: 1355-1365, 2013.

5. Stone A, Cowley MJ, Valdes-Mora F, McCloy RA, Sergio CM, Gallego-Ortega D, Caldon CE, Ormandy CJ, Biankin AV, Gee JM, et al: BCL-2 hypermethylation is a potential biomarker of sensitivity to antimitotic chemotherapy in endocrine-resistant breast cancer. Mol Cancer Ther 12: 1874-1885, 2013.

6. Liu XS, Song B, Elzey BD, Ratliff TL, Konieczny SF, Cheng L, Ahmad N and Liu X: Polo-like kinase 1 facilitates loss of Pten tumor suppressor-induced prostate cancer formation. J Biol Chem 286: 35795-35800, 2011.

7. Song B, Liu XS, Rice SJ, Kuang S, Elzey BD, Konieczny SF, Ratliff TL, Hazbun T, Chiorean EG and Liu X: PLK1 phosphorylation of orc2 and hbol contributes to gemcitabine resistance in pancreatic cancer. Mol Cancer Ther 12: 58-68, 2013.

8. Zhang G, Zhang Z and Liu Z: Polo-like kinase 1 is overexpressed in renal cancer and participates in the proliferation and invasion of renal cancer cells. Tumour Biol 34: 1887-1894, 2013.

9. Zhang Z, Zhang G and Kong C: High expression of polo-like kinase 1 is associated with the metastasis and recurrence in urothelial carcinoma of bladder. Urol Oncol 31: 1222-1230, 2013.

10. Factor VM, Seo D, Ishikawa T, Kaposi-Novak P, Marquardt JU, Andersen JB, Conner EA and Thorgeirsson SS: Loss of c-Met disrupts gene expression program required for $\mathrm{G} 2 / \mathrm{M}$ progression during liver regeneration in mice. PLoS One 5: e12739, 2010.

11. Wu ZQ and Liu X: Role for PLK1 phosphorylation of Hbol in regulation of replication licensing. Proc Natl Acad Sci USA 105: 1919-1924, 2008.

12. Tategu M, Nakagawa H, Sasaki K, Yamauchi R, Sekimachi S, Suita Y, Watanabe N and Yoshid K: Transcriptional regulation of human polo-like kinases and early mitotic inhibitor. J Genet Genomics 35: 215-224, 2008.

13. Ito Y, Miyoshi E, Sasaki N, Kakudo K, Yoshida H, Tomoda C, Uruno T, Takamura Y, Miya A, Kobayashi K, et al: Polo-like kinase 1 overexpression is an early event in the progression of papillary carcinoma. Br J Cancer 90: 414-418, 2004.

14. Myatt SS, Kongsema M, Man CW, Kelly DJ, Gomes AR, Khongkow P, Karunarathna U, Zona S, Langer JK, Dunsby CW, et al: SUMOylation inhibits FOXM1 activity and delays mitotic transition. Oncogene 33: 4316-4329, 2014.

15. Ustiyan V, Wang IC, Ren X, Zhang Y, Snyder J, Xu Y, Wert SE, Lessard JL, Kalin TV and Kalinichenko VV: Forkhead box M1 transcriptional factor is required for smooth muscle cells during embryonic development of blood vessels and esophagus. Dev Biol 336: 266-279, 2009.

16. Kalinichenko VV, Lim L, Shin B and Costa RH: Differential expression of forkhead box transcription factors following butylated hydroxytoluene lung injury. Am J Physiol Lung Cell Mol Physiol 280: L695-L704, 2001. 
17. Zhao F, Siu MK, Jiang L, Tam KF, Ngan HY, Le XF, Wong OG, Wong ES, Gomes AR, Bella L, et al: Overexpression of forkhead box protein M1 (FOXM1) in ovarian cancer correlates with poor patient survival and contributes to paclitaxel resistance. PLoS One 9: e113478, 2014.

18. Gormally MV, Dexheimer TS, Marsico G, Sanders DA, Lowe C, Matak-Vinković D, Michael S, Jadhav A, Rai G, Maloney DJ, et al: Suppression of the FOXM1 transcriptional programme via novel small molecule inhibition. Nat Commun 5: 5165, 2014.

19. Jiang L, Wang P and Chen H: Overexpression of FOXM1 is associated with metastases of nasopharyngeal carcinoma. Ups J Med Sci 119: 324-332, 2014.

20. Li XR, Chu HJ, Lv T, Wang L, Kong SF and Dai SZ: miR-342-3p suppresses proliferation, migration and invasion by targeting FOXM1 in human cervical cancer. FEBS Lett 588: 3298-3307, 2014

21. Ning Z, Wang A, Liang J, Xie Y, Liu J, Feng L, Yan Q and Wang Z: USP22 promotes the G1/S phase transition by upregulating FOXM1 expression via $\beta$-catenin nuclear localization and is associated with poor prognosis in stage II pancreatic ductal adenocarcinoma. Int J Oncol 45: 1594-1608, 2014.

22. Fu Z, Malureanu L, Huang J, Wang W, Li H, van Deursen JM, Tindall DJ and Chen J: Plk1-dependent phosphorylation of FOXM1 regulates a transcriptional programme required for mitotic progression. Nat Cell Biol 10: 1076-1082, 2008.
23. Liu D, Zhang Z and Kong CZ: High FOXM1 expression was associated with bladder carcinogenesis. Tumour Biol 34: 1131-1138, 2013.

24. Ando K, Ozaki T, Hirota T and Nakagawara A: NFBD1/MDC1 is phosphorylated by PLK1 and controls G2/M transition through the regulation of a TOPOII $\alpha$-mediated decatenation checkpoint. PLoS One 8: e82744, 2013.

25. Pezuk JA, Brassesco MS, Morales AG, de Oliveira JC, de Paula Queiroz RG, Machado HR, Carlotti CG Jr, Neder L, Scrideli CA and Tone LG: Polo-like kinase 1 inhibition causes decreased proliferation by cell cycle arrest, leading to cell death in glioblastoma. Cancer Gene Ther 20: 499-506, 2013.

26. Zhang J, Yuan C, Wu J, Elsayed Z and Fu Z: Polo-like kinase 1-mediated phosphorylation of Forkhead box protein M1b antagonizes its SUMOylation and facilitates its mitotic function. J Biol Chem 290: 3708-3719, 2015.

27. Wang R, Song Y, Xu X, Wu Q and Liu C: The expression of Nek7, FoxM1, and PLK1 in gallbladder cancer and their relationships to clinicopathologic features and survival. Clin Transl Oncol 15: 626-632, 2013

28. Dibb M, Han N, Choudhury J, Hayes S, Valentine H, West C, Ang YS and Sharrocks AD: The FOXM1-PLK1 axis is commonly upregulated in oesophageal adenocarcinoma. Br J Cancer 107: 1766-1775, 2012. 